\title{
Macrophages as Key Players during Adipose Tissue- Liver Crosstalk in Nonalcoholic Fatty Liver Disease
}

\author{
Hannelie Korf, $\mathrm{PhD}^{1}$ Markus Boesch, $\mathrm{MS}^{1} \quad$ Lore Meelberghs, $\mathrm{MS}^{1} \quad$ Schalk van der Merwe, MD, $\mathrm{PhD}^{1,2}$ \\ ${ }^{1}$ Laboratory of Hepatology, CHROMETA Department, KU Leuven, \\ Leuven, Belgium \\ 2 Department of Gastroenterology and Hepatology, UZ Leuven, \\ Leuven, Belgium \\ Semin Liver Dis 2019;39:291-300. \\ Address for correspondence Hannelie Korf, PhD, Laboratory of \\ Hepatology, CHROMETA Department, KU Leuven, Leuven 3000 , \\ Belgium (e-mail: hannelie.korf@kuleuven.be). \\ Schalk van der Merwe, MD, PhD, Laboratory of Hepatology, \\ CHROMETA Department, KU Leuven, Leuven 3000, Belgium \\ (e-mail: schalk.vandermerwe@uzleuven.be).
}

\begin{abstract}
Keywords

- nonalcoholic fatty liver disease

- macrophages

- adipose tissue inflammation

- metabolism

Nonalcoholic fatty liver disease (NAFLD) is the most common cause of chronic liver disease in Western countries that could lead to serious health problems including liver failure, cancer, or death. The term NAFLD includes a spectrum of disease states with histological features ranging from simple steatosis to nonalcoholic steatohepatitis (NASH). A key aspect within this research field is the identification of pathogenic factors that trigger inflammation, thus fueling the transition from nonalcoholic fatty liver to NASH. These inflammatory triggers may originate from within the liver as a result of innate immune cell activation and/ or hepatocyte injury. Additionally, they may originate from other sites such as adipose tissue or the intestinal tract. In the current review, the authors will primarily focus on events within adipose tissue which may be of importance in triggering the disease progression. They specifically focus on the role of adipose tissue macrophages during NAFLD pathogenesis and how microenvironmental factors may shape their metabolic profile. They further dissect how redirecting the macrophage's metabolic profile alters their immunological functions. Finally, they discuss the opportunities and challenges of targeting macrophages to interfere in disease progression.
\end{abstract}

Nonalcoholic fatty liver disease (NAFLD) is now one of the most common causes of chronic liver disease in both children and adults and the disease is predicted to become the most important indication for liver transplantation during the next decade. ${ }^{1}$ NAFLD is strongly associated with obesity and metabolic syndrome, and similar to these conditions, the incidence and prevalence of NAFLD are increasing to epidemic proportions. ${ }^{2,3}$ The early stages of NAFLD are hallmarked by accumulation of lipids in hepatocytes (hepatic steatosis). The majority of patients with simple steatosis will not progress to more severe liver disease. However, for reasons incompletely understood, a subset of patients will develop superimposed hepatic inflammation which is referred to as nonalcoholic steatohepatitis (NASH). ${ }^{4}$ Importantly, once NASH is established patients may further progress to cirrhosis and hepatocellular carcinoma. ${ }^{5}$ Currently, there are no approved pharmacological therapies that have been shown to be effective in NASH. The development of novel strategies for NASH treatment will rely on the identification and targeting of key pathogenic pathways. ${ }^{6}$

Although the pathogenesis of NASH is complex and partially unknown, it likely encompasses multiple exogenous as well as endogenous hits resulting in the propagation of liver disease. ${ }^{7}$ In the liver, the excess amounts of circulating fatty acids and carbohydrates result in the accumulation of toxic lipids, oxidative- and ER-stress responses, and eventually hepatocyte death. ${ }^{8} \mathrm{~A}$ high-fat diet or nutrient overload may also trigger qualitative and quantitative changes in gut microbiota that may increase intestinal permeability and translocation of bacterial products to reach the liver through the portal vein. ${ }^{9}$ The continuous exposure to danger-associated molecular patterns (DAMPs) released from necrotic liver cells and pathogen-associated molecular patterns (PAMPs) originating from the gut may sustain and amplify inflammatory events ultimately leading to fibrosis and cirrhosis development. ${ }^{10,11}$ 
The onset of dyslipidemia and inflammation in the liver is closely linked to early events occurring in the adipose tissue. ${ }^{12}$ In both mice and human subjects, the recruitment of macrophages within the adipose tissue compartment has been associated with the development of insulin resistance and steatohepatitis. ${ }^{13,14}$ Conversely, ablation of adipose tissue macrophages, surgical removal of adipose tissue, or inhibiting peroxisome proliferator-activated receptor gamma (PPAR $r$ ) pathways in mice normalized insulin sensitivity and partially reversed liver inflammation..$^{15-17}$ We and others have shown that $\mathrm{CD} 11 \mathrm{c}^{+} \mathrm{CD}_{206}{ }^{+}$and $\mathrm{CCR} 2^{+}$macrophages infiltrate visceral adipose tissue, and are associated with increased production of inflammatory cytokines in NASH. ${ }^{18-20}$ Interestingly, adipose tissue inflammation also preceded the appearance of inflammation in the liver, suggesting that disease-initiating triggers originate from adipose tissue rather than the liver. ${ }^{18,20}$ Notably, while also other immune cells play a role in adipose tissue inflammation, ${ }^{21,22}$ for the purpose of this review we specifically focus on the role of adipose tissue macrophages during NAFLD progression. We further dissect their immunological and metabolic profiles, their interaction with adipocytes as well as their plethora of secreted factors that may fuel inflammation in the liver. Finally, we discuss the translational potential of rewiring the functional or metabolic status of adipose-tissue macrophages.

\section{Molecular Events Triggering Macrophages within the Adipose Tissue Compartment}

During pathological conditions such as NAFLD where chronic overnutrition prevails, the size and number of adipocytes increase to compensate for the excess lipid availability. However, this containment mechanism may ultimately fail leading to adipose tissue dysfunction, dyslipidemia, and insulin resistance. The considerable lipid burden within adipocytes triggers intracellular endoplasmic reticulum (ER) stress mechanisms, which culminate in cell death and the release of lipid contents and cellular debris. ${ }^{8}$ Consequently, tissue resident macrophages are exposed to a variety of triggers such as toxic lipids, oxidative radicals, adipokines, nucleic acids, exosomes, and DAMPs derived from dying cells, and combined these biomolecules create a complex microenvironment that initiates macrophage activation..$^{10,23}$ For example, macrophages surrounding necrotic adipocytes within crown-like structures can become activated following engulfment of necrotic debris. ${ }^{24-26}$ Additionally, danger signals such as high mobility group box protein 1 (HMGB1) can be recognized by toll-like receptors (TLRs) or P2X purinoceptor 7 (P2RX7) on the surface of macrophages, leading to the activation of inflammasomes. ${ }^{27-29}$ Alternatively, toxic lipid compounds can signal macrophage activation and production of proinflammatory mediators by activating intracellular pathways involving key transcription factors such as c-Jun N-terminal kinase (JNK), activator protein 1 (AP-1), and nuclear factorкВ (NF-кB). ${ }^{8}$ Furthermore, extracellular alarmins (e.g., S100A8 and S100A9) can promote human macrophage-mediated inflammation through the receptor advanced glycation endproducts (RAGE) and TLR4-dependent pathways. ${ }^{30,31}$ Finally,
microRNA-155 in adipose-derived microvesicles has been demonstrated to induce macrophage activation, chronic inflammation, and local insulin resistance in a murine model. ${ }^{32}$ An overview of mechanisms that potentiate macrophagemediated inflammation is depicted in - Fig. 1 and has also been reviewed elsewhere. ${ }^{8,10,13,14,21,33}$

Stressed or dying adipocytes also contribute to the recruitment of monocytes/macrophages through the release of chemokines. Although several chemokines have been implicated in this process, ${ }^{34,35} \mathrm{C}-\mathrm{C}$ motif chemokine ligand 2 (CCL2) and its receptor CCR2 seem to play a prominent role ( $\mathbf{- F i g . ~ 1 )})^{36}$ Hereto, the absence of CCR2-blunted macrophage infiltration in an experimental model improved insulin sensitivity and hepatic steatosis. ${ }^{37}$ Conversely, transgenic expression of CCL2 in murine adipose tissue promoted macrophage recruitment and obesity-induced insulin resistance. ${ }^{36}$ These studies establish a prominent role for chemokine-driven recruitment of monocytes/macrophages to adipose tissue. However, whether adipocytes also actively participate in the retention of macrophages to the site of inflammation is less well characterized. Interestingly, a recent report demonstrated that adipose tissue inflammation was dependent on the physical interaction of integrin $\alpha 4$ on macrophages and vascular cell adhesion molecule 1 (VCAM-1), its counter-receptor on adipocytes in a mouse model. ${ }^{38}$ This adhesive interaction resulted in the upregulation of extracellular-signal-regulated kinase (ERK) signaling and the promotion of insulin resistance in adipocytes. ${ }^{38}$ This study shed new light on the extent by which adipocytes sustain adipose tissue inflammation. Additionally, netrin-1 has recently been identified as a macrophage retention molecule ( - Fig. 1) $\cdot{ }^{39}$ Finally, also the production of macrophage migration inhibitor factor (MIF) could be potentially important in both recruiting and retention of macrophages to the adipose tissue site..$^{40,41}$ Combination, recruitment, retention, and activation of macrophages perpetuate a vicious loop of events leading to exacerbated inflammation. ${ }^{42}$

\section{Elucidating the Spectrum of Adipose Tissue Macrophage Phenotypes}

Macrophages are highly versatile cells, with key functions in the initiation as well as resolution of inflammation. ${ }^{43}$ Such functions include phagocytosis of apoptotic/necrotic cells and pathogens, elaboration of immune-effector molecules and growth factors, and remodeling of the extracellular matrix. ${ }^{44}$ Furthermore, they are equipped with a wide range of surface and nuclear receptors to appropriately scale the molecular threat in their microenvironment and can respond accordingly. ${ }^{45}$ If one considers the diversity of signals that these cells encounter in vivo, it is plausible that they exist as a continuum of different activation statuses. ${ }^{43}$ Nevertheless, based on in vitro studies, the extremes of this continuum have been classified in two main groups, namely classically activated/proinflammatory (M1-like) and alternatively activated/anti-inflammatory (M2-like) macrophages. ${ }^{46,47}$ The M1-like macrophage phenotype is driven by proinflammatory mediators such as lipopolysaccharide (LPS) and interferon gamma (IFNY) and is characterized by increased production of proinflammatory 


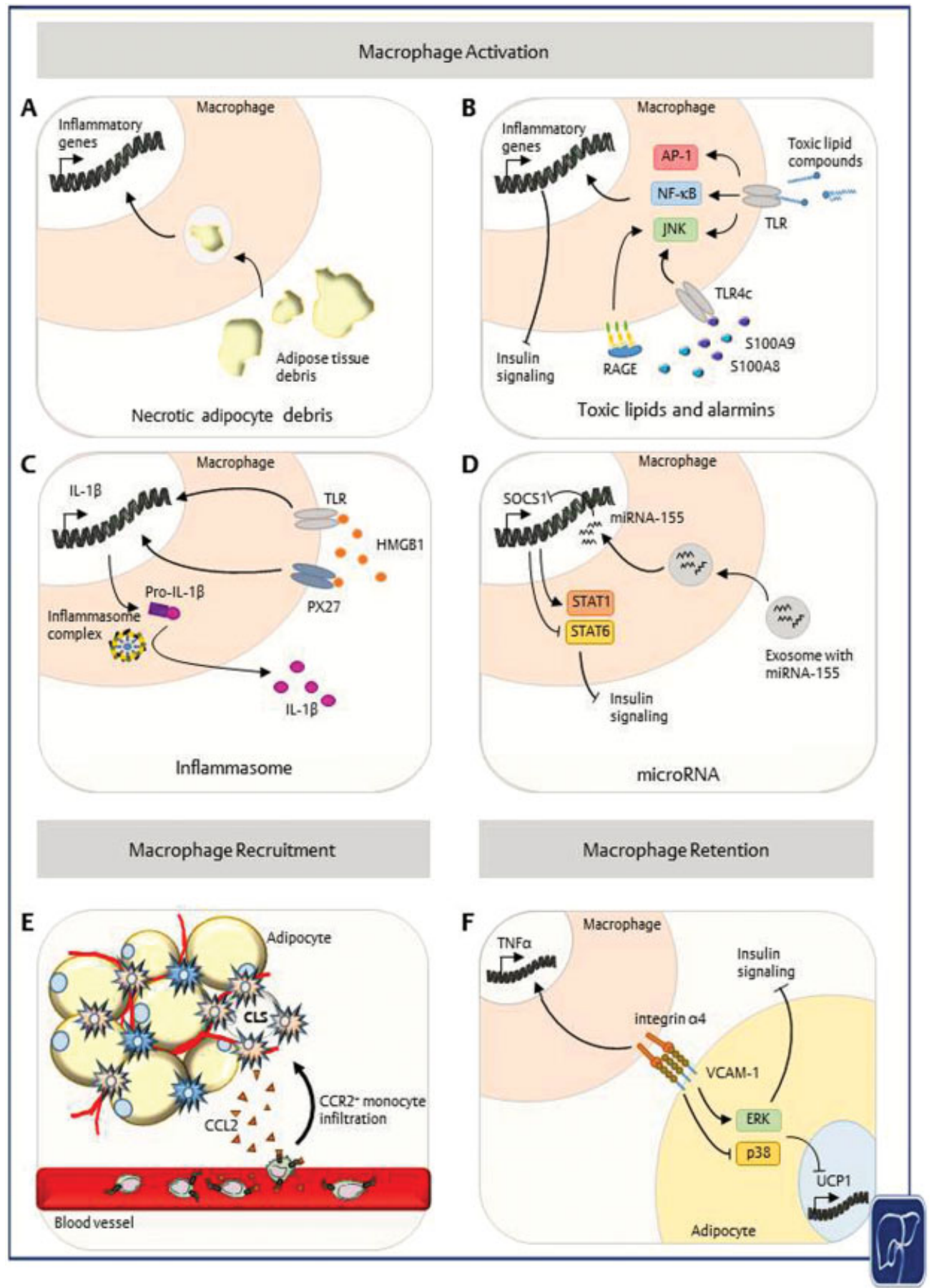

Fig. 1 Molecular mechanism leading to macrophage activation, recruitment, and retention leading to exacerbated adipose tissue inflammation. Macrophages are exposed to several biomolecules initiating their activation (A-D). (A) Necrotic adipocyte debris is engulfed by the surrounding macrophages causing increased expression of proinflammatory genes. (B) Binding of toxic lipid compounds and several alarmins such as S100A8 and S100A9 to TLRs and RAGE elevates the expression of proinflammatory genes via AP-1-, NF-KB-, and JNK-mediated signaling and thereby worsening insulin resistance. (C) Binding of the danger signal HMGB1 to TLRs and P2RX7 can activate the inflammasome complex which will cleave pro-IL-1 $\beta$ into IL-1 $\beta$. (D) Lastly, adipose tissue-derived exosomes contain elevated levels of miRNA-155 which will bind the SOCS1 promoter and inhibit its transcription. This leads to increased STAT1 and decreased STAT6 signaling causing defective insulin signaling. Mechanism triggering macrophage recruitment (E). Necrotic adipocytes produce $\mathrm{CCL} 2$ leading to recruitment of $\mathrm{CCR2}^{+}$monocytes from the circulation into the adipose tissue. These monocytes differentiate to proinflammatory macrophages surrounding the dying adipocytes forming CLS. Mechanisms promoting macrophage retention at the site of inflammation (F). The physical interaction of integrin $\alpha 4$ on macrophages with VCAM-1 on adipocytes causes increased ERK signaling and decreased p38 signaling leading to inhibition of the UCP1 gene and worsening of insulin signaling. Moreover, TNFa transcription in the macrophage is stimulated because of this cell-cell adhesion. AP-1, activator protein 1; CCL/R2, C-C motif chemokine ligand/receptor 2; CLS, crown-like structures; ERK, extracellular-signal-regulated kinase; HMGB1, high mobility group box protein 1; IL-1 $\beta$, interleukin-1 1 ; JNK, c-Jun N-terminal kinases; miRNA, microRNA; NF-KB, nuclear factor-kB; P2RX7, P2X purinoceptor 7; RAGE, receptor for advanced glycation end-products; SOCS1, suppressor of cytokine signaling 1; STAT, signal transducer and activator of transcription; TLR, toll-like receptor; TNF $\alpha$, tumor necrosis factor $\alpha$; VCAM-1, vascular cell adhesion molecule 1; UCP1, uncoupling protein 1. 
cytokines, while the M2-like macrophage phenotype is driven by anti-inflammatory cytokines such as interleukin- 4 (IL-4). ${ }^{48}$

Applying this basic classification in the context of NAFLD resulted in the identification of macrophages with an M2like phenotype in lean adipose tissue and their function to maintain insulin sensitivity through the anti-inflammatory actions of IL-10 and signal transducer and activator of transcription 3 (STAT3). ${ }^{25}$ Furthermore, they activate expression of immunosuppressive factors and PPAR $\gamma$ which promote tissue remodeling and resolve inflammation. ${ }^{48}$ They further produce factors such as insulin-like growth factor 1 (IGF1) and macrophages expressing the receptor of IGF1 have been implicated as negative regulators of inflammation. ${ }^{49}$ Along the same line of investigation, the loss of GPR120, a G protein-coupled receptor with immunoregulatory actions, led to the loss of the inhibitory effect on adipose tissue inflammation which promoted insulin resistance in an experimental mouse model. ${ }^{50}$

M1-like macrophages on the other hand have been described in adipose tissue from NAFLD subjects and they are characterized by the expression of the surface marker CD11c, the secretion of proinflammatory cytokines such as tumor necrosis factor $\alpha$ (TNF $\alpha)$ and IL-6, as well as the generation of reactive oxygen and nitrogen intermediates. ${ }^{18,51}$ In a murine model, macrophages that secrete proinflammatory cytokines induced adipokine dysregulation that impaired insulin action to confer systemic insulin resistance. ${ }^{25}$ The importance of these events in disease pathogenesis has been demonstrated by the fact that ablation of the proinflammatory signaling molecule IKK $\beta$ in murine myeloid cells reduces myeloid cell-mediated inflammation in adipose tissue, resulting in preservation of insulin sensitivity. ${ }^{52}$ Similarly, macrophage-specific deletion of stress-activated protein kinases, JNK, protects against highfat diet-induced obesity and insulin resistance, and reverts M1-like polarization in mice. ${ }^{53}$

Notably, phenotypes of adipose tissue macrophages distinct from classical activation (M1-like) or alternative activation (M2-like) have recently been described. For example, obese adipose tissue macrophages featured increased liposomal biogenesis and lipid catabolism most probably due to chronic lipid overloading in vivo. ${ }^{54}$ Another report described that treating macrophages with a cocktail of glucose, insulin, and fatty acids (palmitate) triggers a metabolically activated state (MMe). ${ }^{55}$ Interestingly, the phenotype of MMe macrophages was driven by the NADPH oxidase 2 complex and they participated in both detrimental and beneficial functions during obesity by promoting inflammatory cytokine production as well as lysosomal exocytosis to adipocytes. ${ }^{56}$ Moreover, another macrophage phenotype termed Mox has also recently been reported in a murine model to be present in lean adipose tissue as a consequence of exposure to truncated oxidized lipids. ${ }^{57}$ Interestingly, these "redox-regulatory" Mox macrophages feature antioxidant gene expression and a quiescent metabolism. ${ }^{57}$ It would be intriguing to further characterize and understand the relevance of these macrophage phenotypes during human disease. Nevertheless, these interesting new findings imply that harnessing macrophage metabolism could represent a promising approach to interfere in NAFLD and associated comorbidities.

\section{Macrophage Metabolic Programs That Govern Immune Function}

Intracellular energy metabolism of macrophages has recently been highlighted as a regulator of their immunological functions. ${ }^{58,59}$ For example, M2-like macrophages, presumed to be present in lean adipose tissue, exhibit a metabolic program that relies on fatty acid oxidation to fuel tricarboxylic acid (TCA) cycle-coupled oxidative phosphorylation. Importantly, the events leading to increased oxidative phosphorylation are orchestrated by the transcription factor STAT6, which in turn induces expression of PPARS, PPARr, and the coactivator protein PGC- $1 \beta{ }^{48,60}$ As demonstrated in a mouse model, M2-like macrophages also maintain insulin sensitivity by the anti-inflammatory actions of IL-10 and STAT3. ${ }^{61}$ Additionally, a role for the mammalian target of rapamycin complex 2 (mTORC2) and interferon regulatory factor 4 (IRF4) signaling axis in M2-like polarization has been demonstrated. ${ }^{62}$ Hereto, the upregulation of both mTORC2 and IRF4 increases glucose-dependent oxidative phosphorylation, which subsequently stimulates the expression of M2-like target genes such as arginase 1 or resistin-like molecule $\alpha$. $^{62}$

Inflammatory-type macrophages, presumed to be present in inflamed adipose tissue, on the other hand are characterized by increased glucose uptake and glycolytic flux, along with impaired oxidative phosphorylation via the TCA cycle. ${ }^{63}$ M1-like macrophages feature an interrupted TCA cycle whereby intermediates such as citrate and succinate accumulate within the cell. ${ }^{64}$ Importantly, these metabolites have been shown to directly affect immune responses. For example, the build-up of citrate in the cytoplasm can promote fatty acid synthesis and production of the antimicrobial metabolite itaconic acid. ${ }^{64}$ Additionally, succinate accumulation leads to stabilization of hypoxia-inducible factor- $1 \alpha$ (HIF-1 $\alpha$ ), a master transcriptional regulator of proinflammatory and glycolytic genes. ${ }^{65}$

However, as mentioned above, we are only starting to grasp the extent of these macrophage phenotypes and their accompanying metabolic signatures especially during NAFLD pathogenesis in an in vivo situation. For instance, it is possible that lipids are excessively engulfed by resident adipose tissue macrophages giving rise to the unique macrophage phenotypes as described above as well as potential novel phenotypes. Key transcription factors that control lipid metabolism in macrophages include PPARs, liver $\mathrm{X}$ receptors (LXRs), CCAAT enhancer-binding proteins (C/EBPs), and sterol regulatory element-binding proteins (SREBPs). ${ }^{66}$ However, how exactly these pathways are dysregulated during NAFLD pathogenesis whereby adipose tissue macrophages fail to cope with the lipid overload remains to be further investigated. Additionally, key molecules that could reverse defective lipid metabolism within these pathways may be highly relevant as disease interventional strategies. 


\section{Promising New Therapeutic Approaches during NAFLD Progression}

\section{Macrophage Recruitment and Activation Status}

There is a major unmet need for effective therapies in fatty liver disease and this is of high clinical relevance considering escalating disease prevalence. Macrophages have key regulatory roles in both inflammation and metabolism, which underscores targeting them for disease intervention (see also overview of new strategies in - Table 1). ${ }^{67}$ In fact, interference with chemokine pathways to restrict proinflammatory monocyte/macrophage recruitment using the CCR2/CCR5 antagonist cenicriviroc is one of the most advanced treatments of NASH-related fibrosis (phase IIb) and a phase III trial is currently ongoing to confirm the efficacy and safety of this drug (the Aurora study). ${ }^{68-70}$ Another key strategy is to promote anti-inflammatory macrophage polarization and consequently also the amelioration of NASH progression by promoting signaling through PPAR pathways. Indeed, recent work in animal models has demonstrated blunted inflammation and reversal of fibrosis is triggered by the PPAR $\alpha /$ PPAR $\gamma$ agonist saroglitazar. ${ }^{71}$ Also in clinical studies, elafibranor, an agonist of PPAR $\alpha$ and $\delta$, has been shown to attenuate inflammation in the liver without adversely affecting fibrosis in patients with NASH. ${ }^{72}$ Alternative approaches include targeting activating signals of myeloid-derived cells in liver disease. In this regard, exciting new research implicated a role for inositol-requiring enzyme $1 \alpha$ (IRE1 $\alpha)$, the

Table 1 Overview of novel therapeutic strategies targeting macrophages during NAFLD

\begin{tabular}{|c|c|c|c|}
\hline Target & Agent & Mechanism of action & Experimental phase \\
\hline \multirow[t]{3}{*}{ Chemokines } & Cenicriviroc & CCR2/CCR5 antagonist reducing M1 and improving fibrosis & Phase III \\
\hline & Maraviroc & $\begin{array}{l}\text { CCR5 antagonist reducing inflammation and improving insulin } \\
\text { sensitivity }\end{array}$ & Preclinical \\
\hline & NIBR2130 & CXCR3 antagonist reducing inflammation & Preclinical \\
\hline IRE1a & $4 \mu 8 \mathrm{C}$ & IRE1a inhibition promoting M2 polarization & Preclinical \\
\hline \multirow[t]{3}{*}{ FXR } & Obeticholic acid & $\begin{array}{l}\text { FXR agonist resulting in shift to M2 macrophages and reduction } \\
\text { of inflammation }\end{array}$ & Phase III \\
\hline & Isoxazoles & FXR agonist improving insulin sensitivity and inflammation & Phase II \\
\hline & EGCG & FXR agonist reducing oxidative stress, inflammation, and fibrosis & Preclinical \\
\hline TGR5/FXR & INT-767 & TGR5/FXR agonist promoting M2 polarization & Preclinical \\
\hline \multirow[t]{4}{*}{ PPAR } & Saroglitazar & $\begin{array}{l}\text { PPAR } \alpha \text { and PPARy agonist, leading to increase of } M 2 \\
\text { macrophages }\end{array}$ & Phase III \\
\hline & Pemafibrate & $\begin{array}{l}\text { PPAR } \alpha \text { and PPAR } \delta \text { agonist modulating lipid turnover and } \\
\text { reducing inflammation }\end{array}$ & Phase II \\
\hline & Elafibranor & $\begin{array}{l}\text { PPAR } \alpha \text { and PPARס agonist improving insulin sensitivity and } \\
\text { inflammation }\end{array}$ & Phase II \\
\hline & L-165041 & $\begin{array}{l}\text { PPAR } \alpha \text { and PPAR } \delta \text { agonist improving insulin sensitivity and } \\
\text { inflammation }\end{array}$ & Preclinical \\
\hline ASK1 & Selonsertib & $\begin{array}{l}\text { ASK1 inhibitor improving fibrosis and reducing inflammation by } \\
\text { increasing M2 polarization }\end{array}$ & Phase III \\
\hline SKY & R406-PLGA & $\begin{array}{l}\text { R406-PLGA nanoparticle, inhibiting SKY pathway in } \\
\text { macrophages, improving fibrosis and inflammation }\end{array}$ & Preclinical \\
\hline \multirow[t]{3}{*}{ microRNA } & AntimiR-221/222 & $\begin{array}{l}\text { Inhibition of microRNA- } 221 \text { and microRNA-222, improving } \\
\text { fibrosis, and reducing inflammation }\end{array}$ & Preclinical \\
\hline & MRG-201 (miRagen) & microRNA-29 mimic, improving fibrosis & Phase I \\
\hline & $\begin{array}{l}\text { Lac-PDMAEMA/ } \\
\text { miR-146b mimic }\end{array}$ & $\begin{array}{l}\text { Targeted delivery of micro-RNA146b mimic via nanoparticles to } \\
\text { hepatocytes, reducing inflammation }\end{array}$ & Preclinical \\
\hline mTORC1 & Rapamycin & $\begin{array}{l}\text { mTORC1 inhibition improves inflammation and insulin } \\
\text { sensitivity }\end{array}$ & Preclinical \\
\hline NOTCH1 & Curcumin & $\begin{array}{l}\text { NOTCH1 inhibition improves inflammation and insulin } \\
\text { sensitivity }\end{array}$ & Preclinical \\
\hline AMPK & Tamoxifen & $\begin{array}{l}\text { AMPK activation inhibits M1 polarization and improves } \\
\text { inflammation }\end{array}$ & Preclinical \\
\hline SIRT1/2 & SRT1720 & $\begin{array}{l}\text { SIRT1/2 activation improves inflammation by inhibiting NF-KB } \\
\text { signaling }\end{array}$ & Preclinical \\
\hline
\end{tabular}

Abbreviations: AMPK, 5' AMP-activated protein kinase; ASK1, apoptosis signal-regulating kinase 1; FXR, farnesoid X receptor; IRE1 $\alpha$ inositol-requiring enzyme $1 \alpha$; mTORC, mammalian target of rapamycin complex; NF-kB, nuclear factor-kB; PPAR, peroxisome proliferator-activated receptor. 
upstream regulator of a part of the unfolded protein response, in aggravating inflammation and the obesityassociated symptoms by reprogramming macrophage function. ${ }^{73}$ Mechanistically, IRE1 $\alpha$ plays a role in suppressing IRF4 and KLF4, both key transcription factors promoting M2like polarization. Consequently, ablation of IRE1 $\alpha$ in mice attenuated the shift towards the proinflammatory M1-like phenotype, and simultaneously promoted the anti-inflammatory M2-like phenotype. ${ }^{73}$ Similarly, a dual agonist for Gprotein coupled $\mathrm{BA}$ receptor 5 and farnesoid $\mathrm{X}$ receptor (TGR5/FXR) triggered elevated frequencies of anti-inflammatory monocytes/macrophages and protected against steatohepatitis in a murine model. ${ }^{74}$ Moreover, the semisynthetic bile acid analogue obeticholic acid is a strong FXR agonist with promising results from an early phase clinical trial in patients with NASH. ${ }^{75}$

\section{Shifting the Macrophage Metabolic Program}

Additionally, approaches aiming at metabolic rewiring of macrophages to regulate their immune function may be of equal importance and this concept is already well established in other disease fields. With relevance to NAFLD, a promising study demonstrated that inhibition of the key metabolic regulator, mTORC1, improved high-fat dietinduced steatohepatitis through modulation of lipid metabolism, macrophage polarization, inflammatory response, and autophagy. ${ }^{76}$ Mice with a selective deficiency of mTORC1 in macrophages portrayed a predominantly M2-like phenotype, reduced ER stress, reduced inflammation in the liver, and improved insulin sensitivity. ${ }^{76}$ Another interesting study also implicates a role for the NOTCH1 pathway in promoting mitochondrial oxidative phosphorylation and reactive oxygen species as well as the expression of M1-related genes. Importantly, conditional deficiency of NOTCH1 in myeloid cells attenuated M1-like activation of hepatic macrophages and inflammation in a murine model of alcoholic steatohepatitis. ${ }^{77}$ Mechanistically, ligand binding to the Notch receptor triggers proteolytic cleavage of its receptor, resulting in the release of Notch intracellular domain (NICD). In turn, NICD translocates to the nucleus and binds to recombining binding protein suppressor of hairless (RBP-J), resulting in the release of IRF8 and NF-KB. Moreover, the sedoheptulose kinase of the pentose phosphate pathway, carbohydrate kinase-like (CARKL) protein, plays a role in regulating a metabolic switch toward glycolysis in M1-like macrophages. ${ }^{78}$ Consequently, knock down of CARKL removed the negative regulation on glycolysis and produced a clear macrophage phenotype. In turn, overexpression of CARKL blunted the expression of IL- 6 and TNF $\alpha$ while augmenting IL-10. ${ }^{78}$ Finally, 5' AMP-activated protein kinase (AMPK) was shown to induce an anti-inflammatory phenotype in macrophages through inhibiting proinflammatory polarization and crucially contributes to immune function in macrophages. $^{79}$

\section{Epigenetic Regulation and Innate Immune Memory} Another concept that opens a window of opportunity for new interventions stems from studies demonstrating that epige- netic mechanisms can regulate macrophage function by imprinting them with a memory response towards future stimuli. ${ }^{80,81}$ Notably the concept of innate immune memory has been established in infection studies whereby macrophages modify their histone acetylation and methylation traits, to become either 'trained' or 'tolerant' upon exposure to subsequent stimulation. ${ }^{82}$ For example, during trained memory a metabolic switch from oxidative phosphorylation to aerobic glycolysis occurs through activation of the mTORHIF1 $\alpha$ pathway. Mechanistically, this was achieved through increased trimethylated histone H3K4 and acetylated histone H3K27 in the promoter region of the main mTOR-target gene and thereby permitting its transcription. ${ }^{83}$ In contrast, macrophages that exhibit endotoxin tolerance upon TLR4 activation undergo a metabolic switch from glycolysis to oxidative phosphorylation through activation of the histone deacetylases sirtuin-1 and sirtuin-6 (SIRT1/6) and consequent inhibition of inflammatory gene transcription. ${ }^{84}$ Notably, these pathways are potentially targetable since inhibitors of the histone deacetylases SIRT1/2 have been shown to have the capacity to reverse immune paralysis in experimental sepsis models. ${ }^{85,86}$ Importantly, a recent study also demonstrated that macrophages can exhibit a molecular memory after digesting apoptotic cells. ${ }^{87}$ The mechanism thereof was dependent on JNK-induced upregulation of the damage signal Draper leading to imprinting of an antiinflammatory response following corpse engulfment. ${ }^{87}$ Further support of the concept that innate memory can also be induced by danger signals within a sterile inflammation environment stems from studies within the atherosclerosis field. For example, monocytes exposed to oxidized low-density lipoproteins exhibit increased expression of inflammatory cytokines following secondary challenge with triggers of activation, and this effect was reversed by treatment with a methylation inhibitor. ${ }^{88}$ Whether such a molecular memory imprinting of tolerant or anti-inflammatory macrophages can be achieved during NAFLD might be highly relevant to investigate in the future.

\section{Adipose Tissue-Liver Crosstalk}

The key question in the disease pathogenesis of NASH is to understand the interorgan relationship of adipose tissue inflammation, which drives the development of steatohepatitis in the liver. Notably, it is well established that adipose tissue engages in crosstalk with the liver influencing whole body metabolism and insulin resistance. For example, it is known that signaling molecules (e.g., microRNAs, adipokines, lipotoxic molecules, cytokines/chemokines, DAMPs, and metabolites) released from the adipose tissue into the portal vein can potentially trigger inflammation in the liver. Of note, also molecules derived from the intestinal compartment can have similar repercussions on pathological events in the liver; however, this will not be addressed here as this aspect has been extensively reviewed elsewhere. ${ }^{33,89}$ The key issue is to define what types of molecule direct diseaserelevant events at a distant site such as the liver, as well as the mechanistic insight into the targeted molecular pathways. 


\section{Toxic Lipids}

During obesity, adipocytes lose the capacity to efficiently store triglyceride, leading to free fatty acid release into the circulation and drainage to the liver. ${ }^{8}$ Hepatic fatty acid availability induces accumulation of lipid intermediates, especially diacylglycerol, which activates specific isoforms of protein kinase $C$ and hampers insulin receptor activation and insulin-stimulated glycogen synthesis. ${ }^{90}$ Establishment of insulin resistance further increases the flux of substrates that promote lipogenesis and gluconeogenesis (-Fig. 2). ${ }^{8}$ Furthermore, free fatty acids activate transcription of SREBP-1c further promoting lipogenesis and hepatic steatosis. ${ }^{91}$ Additionally, some lipid moieties such as palmitic acid, ceramides and lysophosphatidylcholine can also hamper the function of intracellular organelles such as the ER and the mitochondria, triggering cellular stress or even hepatocyte death. ${ }^{8}$ Besides their effects on hepatocytes, lipotoxic agents can influence the activation status of hepatic macrophages and Kupffer cells (KCs; - Fig. 2). For example, palmitic acid can activate TLR2 and TLR4 in macrophages, resulting in upregulated expression of proinflammatory cytokines, via NF-кB, AP-1, and activation of the JNK pathway. ${ }^{92}$

\section{miroRNAs}

A typical example of powerful adipose-tissue-derived messenger molecules that regulate gene expression in other organs such as the liver is microRNAs (miRNAs). miRNAs are snips of noncoding RNA produced intracellularly and are secreted into the circulation either as free entities or packaged into small vesicles called exosomes. ${ }^{93}$ They mediate their effects either by mRNA cleavage, translational repression, or mRNA destabilization following binding to target transcript sequences ( $\mathbf{F i g . 2}$ ). ${ }^{93}$ As proof of concept, the ability of adipose-tissue derived miRNA to directly regulate expression of FGF21 in the liver has recently been demonstrated in a mouse model. ${ }^{94,95}$ More recent studies also implicate a role for miRNAs in disease progression from simple steatosis to NASH, further highlighting their relevance as potential therapeutic targets. For example, adipose tissue macrophages from obese animals secrete miRNA-containing exosomes that can influence local and systemic insulin resistance. In contrast, treatment of obese recipients with adipose tissue macrophage-derived exosomes from lean mice leads to a significant improvement in insulin sensitivity. Finally, a role for miRNA-155 in inhibiting insulin signaling has been implicated through a mechanism related to suppression of its target gene, PPAR ${ }^{96}$ Furthermore, also miRNA-221 and miRNA-222 have been shown to be elevated in NAFLD and importantly, antimiRNAs of miRNA-221/222 inhibited fibrosis and improved insulin signaling in a preclinical NAFLD model. ${ }^{97}$

\section{Adipokines}

Leptin released by the adipose tissue compartment typically augments hepatic transforming growth factor- $\beta$ (TGF- $\beta$ ) and promotes the fibrotic response, a process orchestrated by the interplay between KCs and stellate cells (- Fig. 2) ${ }^{98}$ Leptin can also promote acute inflammation by triggering the release of KC-derived TNF $\alpha$ and trigger KC activation through oxidative stress mechanisms (e.g., iNOS and NADPH oxidase). ${ }^{98-100}$ Leptin also prompted elevated expression of the LPS receptor CD14 through triggering STAT3 signaling in KCs and thereby increased their responsiveness towards danger signals. ${ }^{101}$ On the other hand, adiponectin could play a more protective role through decreasing $\mathrm{KC}$ sensitivity to danger signals or exhibiting an antiproliferative effect on hepatic stellate cells. ${ }^{102} \mathrm{An}$ extensive overview of all adipokines and their downstream functions has been recently documented. ${ }^{23}$

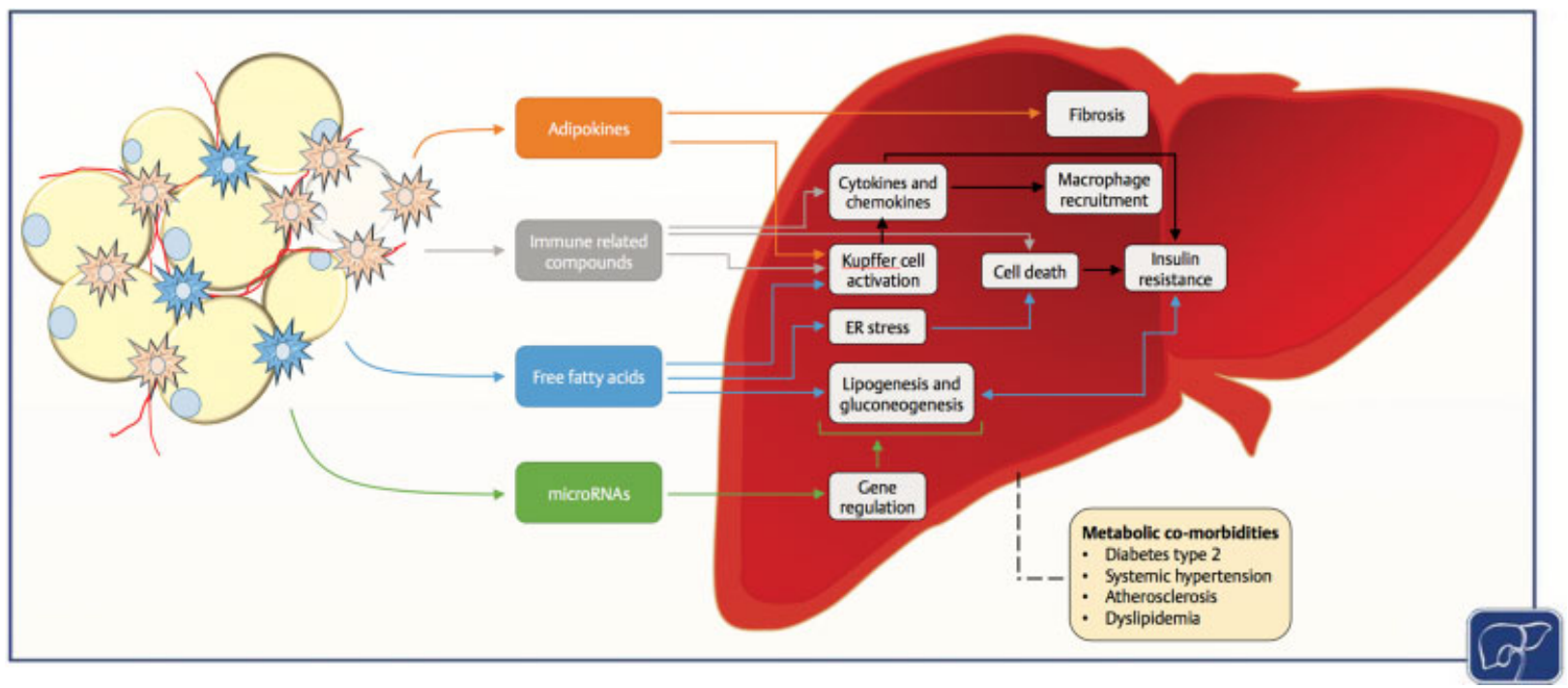

Fig. 2 Mechanisms involved in adipose tissue-liver crosstalk during NAFLD. Inflamed adipose tissue secretes a diverse array of molecules, which transfer to the liver via the portal circulation, causing a cascade of inflammatory, metabolic, and profibrotic events. For example, the secretion of adipokines exhibits a profibrotic effect and activates KCs. Immune compounds can trigger recruitment of innate immune effector cells and their activation. Toxic-free fatty acid moieties can initiate ER stress and cell death pathways and can contribute to KC activation. The flux of lipid species to the liver further increases intrahepatic lipogenesis and gluconeogenesis causing hepatic insulin resistance. Combined, these effects can lead to a metabolic dysregulation at a systemic level, thereby promoting the development of comorbidities such as diabetes type 2, dyslipidemia, systemic hypertension, and atherosclerosis. MicroRNAs are able to regulate gene expression in all the different hepatic cell types. ER, endoplasmic reticulum; KCs, Kupffer cells; NAFLD, nonalcoholic fatty liver disease. 


\section{Immune-Related Compounds}

The adipose tissue compartment also secretes danger signals or inflammatory parameters into the portal circulation that can promote pathogenic events in the liver (-Fig. 2). For example, adipose tissue-derived S100A8 and S100A9 can potentially trigger local and ectopic macrophage activation through TLR4 and NLRP3 signaling and IL-1 $\beta$ production. $^{103}$ Similarly, TNF $\alpha$ secreted from adipose tissue macrophages induces cell death via JNK pathways and promotes KC activation, further perpetuating inflammation in the liver. ${ }^{104}$ Interestingly, recent animal studies also highlight that macrophages recruited to obese visceral adipose tissue contribute to neutrophil recruitment in the liver, and consequently, the development and progression of NASH. ${ }^{105}$ The data suggest that the elevated hepatic neutrophilic inflammation was potentially mediated by an increase in the neutrophil chemotactic factors CXCL14 and CXCL16 by adipose tissue macrophages. ${ }^{105}$ Finally, the release of plasminogen activator inhibitor-1, a serine protease inhibitor that suppresses the breakdown of blood clots, can promote shunting of free fatty acids to ectopic sites such as the liver and contributes to systemic insulin resistance and the thrombosis risk. ${ }^{106}$ Combined, these factors may not only promote disease progression of NAFLD but also the pathogenesis of cardiovascular diseases and type 2 diabetes typically associated with the disease (-Fig. 2).

\section{Conclusion}

Increasing evidence highlights the close association between macrophage function and metabolism and its importance during NAFLD. As outlined above, approaches such as functional or metabolic rewiring and targeting key epigenetic regulators within macrophages could potentially hold promise in designing novel therapeutic strategies. Of note, the availability of small molecules capable of manipulating metabolic and epigenetic traits may proof useful in these studies. Furthermore, key techniques such as single-cell RNA sequencing, single-nucleus sequencing, and/or single-molecule mRNA fluorescent in situ hybridization will be crucial in unraveling these complex mechanisms. In fact, highly exciting new research using single-cell RNA sequencing has revealed distinct populations of liver-resident monocytes/ macrophages in mouse and human tissue. ${ }^{107,108}$ It is plausible that these intrahepatic macrophages are receptive to signals and metabolites released from the adipose tissue and therefore play an equally important role in the crosstalk between the adipose tissue and the liver. ${ }^{33}$ It will therefore be crucial for future studies to map these interorgan transcriptome signatures at a single-cell level, not only in a naïve condition, but also during the different stages of NAFLD. The latter may reveal molecular mechanisms that could potentially be exploited to combat this disease with its epidemic proportions.

\section{Conflicts of Interest}

None.

\section{References}

1 Younossi Z, Henry L. Contribution of alcoholic and nonalcoholic fatty liver disease to the burden of liver-related morbidity and mortality. Gastroenterology 2016;150(08):1778-1785

2 Lallukka S, Yki-Järvinen H. Non-alcoholic fatty liver disease and risk of type 2 diabetes. Best Pract Res Clin Endocrinol Metab 2016;30(03):385-395

3 Wong RJ, Aguilar M, Cheung R, et al. Nonalcoholic steatohepatitis is the second leading etiology of liver disease among adults awaiting liver transplantation in the United States. Gastroenterology 2015;148(03):547-555

4 Rinella ME, Sanyal AJ. Management of NAFLD: a stagebased approach. Nat Rev Gastroenterol Hepatol 2016;13(04): 196-205

5 Adams LA, Anstee QM, Tilg H, Targher G. Non-alcoholic fatty liver disease and its relationship with cardiovascular disease and other extrahepatic diseases. Gut 2017;66(06):1138-1153

6 Musso G, Cassader M, Gambino R. Non-alcoholic steatohepatitis: emerging molecular targets and therapeutic strategies. Nat Rev Drug Discov 2016;15(04):249-274

7 Tilg H, Moschen AR. Evolution of inflammation in nonalcoholic fatty liver disease: the multiple parallel hits hypothesis. Hepatology 2010;52(05):1836-1846

8 Marra F, Svegliati-Baroni G. Lipotoxicity and the gut-liver axis in NASH pathogenesis. J Hepatol 2018;68(02):280-295

9 Tremaroli V, Bäckhed F. Functional interactions between the gut microbiota and host metabolism. Nature 2012;489(7415):242-249

10 Cai J, Zhang X-J, Li H. Role of innate immune signaling in nonalcoholic fatty liver disease. Trends Endocrinol Metab 2018;29 (10):712-722

11 Fuchs M, Schnabl B. Editors' introduction to the NAFLD and NASH special issue. Dig Dis Sci 2016;61(05):1211-1213

12 Arrese M, Cabrera D, Kalergis AM, Feldstein AE. Innate immunity and inflammation in NAFLD/NASH. Dig Dis Sci 2016;61(05):1294-1303

13 Chawla A, Nguyen KD, Goh YPS. Macrophage-mediated inflammation in metabolic disease. Nat Rev Immunol 2011;11(11):738-749

14 Boutens L, Stienstra R. Adipose tissue macrophages: going off track during obesity. Diabetologia 2016;59(05):879-894

15 Mulder P, Morrison MC, Wielinga PY, van Duyvenvoorde W, Kooistra T, Kleemann R. Surgical removal of inflamed epididymal white adipose tissue attenuates the development of non-alcoholic steatohepatitis in obesity. Int J Obes 2016;40(04):675-684

16 Patsouris D, Li P-P, Thapar D, Chapman J, Olefsky JM, Neels JG. Ablation of CD11c-positive cells normalizes insulin sensitivity in obese insulin resistant animals. Cell Metab 2008;8(04):301-309

17 Mulder P, Morrison MC, Verschuren L, et al. Reduction of obesityassociated white adipose tissue inflammation by rosiglitazone is associated with reduced non-alcoholic fatty liver disease in LDLr-deficient mice. Sci Rep 2016;6(01):31542

18 du Plessis J, van Pelt J, Korf $\mathrm{H}$, et al. Association of adipose tissue inflammation with histologic severity of nonalcoholic fatty liver disease. Gastroenterology 2015;149(03):635.e14-648.e14

19 Wentworth JM, Naselli G, Brown WA, et al. Pro-inflammatory CD11c + CD206+ adipose tissue macrophages are associated with insulin resistance in human obesity. Diabetes 2010;59 (07):1648-1656

20 Stanton MC, Chen S-C, Jackson JV, et al. Inflammatory signals shift from adipose to liver during high fat feeding and influence the development of steatohepatitis in mice. J Inflamm (Lond) 2011;8(01):8

21 Sun S, Ji Y, Kersten S, Qi L. Mechanisms of inflammatory responses in obese adipose tissue. Annu Rev Nutr 2012;32 (01):261-286

22 Cildir G, Akıncilar SC, Tergaonkar V. Chronic adipose tissue inflammation: all immune cells on the stage. Trends Mol Med 2013;19(08):487-500

23 Ouchi N, Parker JL, Lugus JJ, Walsh K. Adipokines in inflammation and metabolic disease. Nat Rev Immunol 2011;11(02):85-97 
24 Gericke M, Weyer U, Braune J, Bechmann I, Eilers J. A method for longterm live imaging of tissue macrophages in adipose tissue explants. Am J Physiol Endocrinol Metab 2015;308(11):E1023-E1033

25 Lumeng CN, Deyoung SM, Bodzin JL, Saltiel AR. Increased inflammatory properties of adipose tissue macrophages recruited during diet-induced obesity. Diabetes 2007;56(01):16-23

26 Lumeng CN, DelProposto JB, Westcott DJ, Saltiel AR. Phenotypic switching of adipose tissue macrophages with obesity is generated by spatiotemporal differences in macrophage subtypes. Diabetes 2008;57(12):3239-3246

27 Pétrilli V, Dostert C, Muruve DA, Tschopp J. The inflammasome: a danger sensing complex triggering innate immunity. Curr Opin Immunol 2007;19(06):615-622

28 Vandanmagsar B, Youm Y-H, Ravussin A, et al. The NLRP3 inflammasome instigates obesity-induced inflammation and insulin resistance. Nat Med 2011;17(02):179-188

29 Wree A, Marra F. The inflammasome in liver disease. J Hepatol 2016;65(05):1055-1056

30 Fassl SK, Austermann J, Papantonopoulou O, et al. Transcriptome assessment reveals a dominant role for TLR4 in the activation of human monocytes by the alarmin MRP8. J Immunol 2015;194 (02):575-583

31 Xia C, Braunstein Z, Toomey AC, Zhong J, Rao X. S100 proteins as an important regulator of macrophage inflammation. Front Immunol 2018;8:1908

32 Zhang Y, Mei H, Chang X, Chen F, Zhu Y, Han X. Adipocyte-derived microvesicles from obese mice induce $M 1$ macrophage phenotype through secreted miR-155. J Mol Cell Biol 2016;8(06):505-517

33 Hundertmark J, Krenkel O, Tacke F. Adapted immune responses of myeloid-derived cells in fatty liver disease. Front Immunol 2018;9:2418

34 Bai Y, Sun Q. Macrophage recruitment in obese adipose tissue. Obes Rev 2015;16(02):127-136

$35 \mathrm{Kim}$ D, Kim J, Yoon JH, et al. CXCL12 secreted from adipose tissue recruits macrophages and induces insulin resistance in mice. Diabetologia 2014;57(07):1456-1465

36 Kanda H, Tateya S, Tamori Y, et al. MCP-1 contributes to macrophage infiltration into adipose tissue, insulin resistance, and hepatic steatosis in obesity. J Clin Invest 2006;116(06):1494-1505

37 Weisberg SP, Hunter D, Huber R, et al. CCR2 modulates inflammatory and metabolic effects of high-fat feeding. J Clin Invest 2006;116(01):115-124

38 Chung K-J, Chatzigeorgiou A, Economopoulou M, et al. A selfsustained loop of inflammation-driven inhibition of beige adipogenesis in obesity. Nat Immunol 2017;18(06):654-664

39 Ramkhelawon B, Hennessy EJ, Ménager M, et al. Netrin-1 promotes adipose tissue macrophage retention and insulin resistance in obesity. Nat Med 2014;20(04):377-384

40 Finucane OM, Reynolds CM, McGillicuddy FC, et al. Macrophage migration inhibitory factor deficiency ameliorates high-fat diet induced insulin resistance in mice with reduced adipose inflammation and hepatic steatosis. PLoS One 2014;9(11): e113369

41 Finucane OM, Reynolds CM, McGillicuddy FC, Roche HM. Insights into the role of macrophage migration inhibitory factor in obesity and insulin resistance. Proc Nutr Soc 2012;71(04):622-633

42 Weisberg SP, McCann D, Desai M, Rosenbaum M, Leibel RL, Ferrante AW Jr. Obesity is associated with macrophage accumulation in adipose tissue. J Clin Invest 2003;112(12):1796-1808

43 Ginhoux F, Schultze JL, Murray PJ, Ochando J, Biswas SK. New insights into the multidimensional concept of macrophage ontogeny, activation and function. Nat Immunol 2016;17(01):34-40

44 Ginhoux F, Jung S. Monocytes and macrophages: developmental pathways and tissue homeostasis. Nat Rev Immunol 2014;14 (06):392-404

45 Blander JM, Sander LE. Beyond pattern recognition: five immune checkpoints for scaling the microbial threat. Nat Rev Immunol 2012;12(03):215-225
46 Mantovani A, Sica A, Sozzani S, Allavena P, Vecchi A, Locati M. The chemokine system in diverse forms of macrophage activation and polarization. Trends Immunol 2004;25(12):677-686

47 Gordon S. Alternative activation of macrophages. Nat Rev Immunol 2003;3(01):23-35

48 Odegaard JI, Ricardo-Gonzalez RR, Goforth MH, et al. Macrophage-specific PPARgamma controls alternative activation and improves insulin resistance. Nature 2007;447(7148):1116-1120

49 Spadaro O, Camell CD, Bosurgi L, et al. IGF1 shapes macrophage activation in response to immunometabolic challenge. Cell Reports 2017;19(02):225-234

50 Oh DY, Talukdar S, Bae EJ, et al. GPR120 is an omega-3 fatty acid receptor mediating potent anti-inflammatory and insulin-sensitizing effects. Cell 2010;142(05):687-698

51 Cho KW, Morris DL, Lumeng CN. Flow cytometry analyses of adipose tissue macrophages. Methods Enzymol 2014;537:297-314

52 Arkan MC, Hevener AL, Greten FR, et al. IKK- $\beta$ links inflammation to obesity-induced insulin resistance. Nat Med 2005;11(02):191-198

53 Han MS, Jung DY, Morel C, et al. JNK expression by macrophages promotes obesity-induced insulin resistance and inflammation. Science 2013;339(6116):218-222

54 Xu X, Grijalva A, Skowronski A, van Eijk M, Serlie MJ, Ferrante AW Jr. Obesity activates a program of lysosomal-dependent lipid metabolism in adipose tissue macrophages independently of classic activation. Cell Metab 2013;18(06):816-830

55 Kratz M, Coats BR, Hisert KB, et al. Metabolic dysfunction drives a mechanistically distinct proinflammatory phenotype in adipose tissue macrophages. Cell Metab 2014;20(04):614-625

56 Coats BR, Schoenfelt KQ Barbosa-Lorenzi VC, et al. Metabolically activated adipose tissue macrophages perform detrimental and beneficial functions during diet-induced obesity. Cell Reports 2017;20(13):3149-3161

57 Serbulea V, Upchurch CM, Schappe MS, et al. Macrophage phenotype and bioenergetics are controlled by oxidized phospholipids identified in lean and obese adipose tissue. Proc Natl Acad Sci U S A 2018;115(27):E6254-E6263

58 O'Neill LAJ, Pearce EJ. Immunometabolism governs dendritic cell and macrophage function. J Exp Med 2016;213(01):15-23

59 Geeraerts X, Bolli E, Fendt S-M, Van Ginderachter JA. Macrophage metabolism as therapeutic target for cancer, atherosclerosis, and obesity. Front Immunol 2017;8:289

60 Chawla A. Control of macrophage activation and function by PPARs. Circ Res 2010;106(10):1559-1569

61 Lumeng CN, Bodzin JL, Saltiel AR. Obesity induces a phenotypic switch in adipose tissue macrophage polarization. J Clin Invest 2007;117(01):175-184

62 Huang SC-C, Smith AM, Everts B, et al. Metabolic reprogramming mediated by the mTORC2-IRF4 signaling axis is essential for macrophage alternative activation. Immunity 2016;45(04): 817-830

63 Kelly B, O'Neill LA. Metabolic reprogramming in macrophages and dendritic cells in innate immunity. Cell Res 2015;25(07): 771-784

64 Jha AK, Huang SCC, Sergushichev A, et al. Network integration of parallel metabolic and transcriptional data reveals metabolic modules that regulate macrophage polarization. Immunity 2015;42(03):419-430

65 Tannahill GM, Curtis AM, Adamik J, et al. Succinate is an inflammatory signal that induces IL- $1 \beta$ through HIF- $1 \alpha$. Nature 2013;496(7444):238-242

66 Remmerie A, Scott CL. Macrophages and lipid metabolism. Cell Immunol 2018;330:27-42

67 Tacke F. Targeting hepatic macrophages to treat liver diseases. J Hepatol 2017;66(06):1300-1312

68 Friedman SL, Ratziu V, Harrison SA, et al. A randomized, placebocontrolled trial of cenicriviroc for treatment of nonalcoholic steatohepatitis with fibrosis. Hepatology 2018;67(05): 1754-1767 
69 Lefebvre E, Moyle G, Reshef R, et al. Antifibrotic effects of the dual CCR2/CCR5 antagonist cenicriviroc in animal models of liver and kidney fibrosis. PLoS One 2016;11(06):e0158156

70 Krenkel O, Puengel T, Govaere O, et al. Therapeutic inhibition of inflammatory monocyte recruitment reduces steatohepatitis and liver fibrosis. Hepatology 2018;67(04):1270-1283

71 Jain MR, Giri SR, Bhoi B, et al. Dual PPAR $\alpha / \gamma$ agonist saroglitazar improves liver histopathology and biochemistry in experimental NASH models. Liver Int 2018;38(06):1084-1094

72 Ratziu V, Harrison SA, Francque S, et al; GOLDEN-505 Investigator Study Group. Elafibranor, an agonist of the peroxisome proliferator-activated receptor- $\alpha$ and $-\delta$, induces resolution of nonalcoholic steatohepatitis without fibrosis worsening. Gastroenterology 2016;150(05):1147.e5-1159.e5

73 Shan B, Wang X, Wu Y, et al. The metabolic ER stress sensor IRE1 $\alpha$ suppresses alternative activation of macrophages and impairs energy expenditure in obesity. Nat Immunol 2017;18(05):519-529

74 McMahan RH, Wang XX, Cheng LL, et al. Bile acid receptor activation modulates hepatic monocyte activity and improves nonalcoholic fatty liver disease. J Biol Chem 2013;288(17): 11761-11770

75 Neuschwander-Tetri BA, Loomba R, Sanyal AJ, et al; NASH Clinical Research Network. Farnesoid X nuclear receptor ligand obeticholic acid for non-cirrhotic, non-alcoholic steatohepatitis (FLINT): a multicentre, randomised, placebo-controlled trial. Lancet 2015;385(9972):956-965

76 Lin C-W, Zhang H, Li M, et al. Pharmacological promotion of autophagy alleviates steatosis and injury in alcoholic and nonalcoholic fatty liver conditions in mice. J Hepatol 2013;58(05): 993-999

$77 \mathrm{Xu}$ J, Chi F, Guo T, et al. NOTCH reprograms mitochondrial metabolism for proinflammatory macrophage activation. J Clin Invest 2015;125(04):1579-1590

78 Haschemi A, Kosma P, Gille L, et al. The sedoheptulose kinase CARKL directs macrophage polarization through control of glucose metabolism. Cell Metab 2012;15(06):813-826

79 Sag D, Carling D, Stout RD, Suttles J. Adenosine 5'-monophosphate-activated protein kinase promotes macrophage polarization to an anti-inflammatory functional phenotype. J Immunol 2008;181(12):8633-8641

80 Netea MG, van der Meer JWM. Trained immunity: an ancient way of remembering. Cell Host Microbe 2017;21(03):297-300

81 Baardman J, Licht I, de Winther MPJ, Van den Bossche J. Metabolic-epigenetic crosstalk in macrophage activation. Epigenomics 2015;7(07):1155-1164

82 Netea MG, Latz E, Mills KHG, O'Neill LAJ. Innate immune memory: a paradigm shift in understanding host defense. Nat Immunol 2015;16(07):675-679

83 Cheng S-C, Quintin J, Cramer RA, et al. mTOR- and HIF- $1 \alpha-$ mediated aerobic glycolysis as metabolic basis for trained immunity. Science 2014;345(6204):1250684

84 Liu TF, Yoza BK, El Gazzar M, Vachharajani VT, McCall CE. NAD+dependent SIRT1 deacetylase participates in epigenetic reprogramming during endotoxin tolerance. J Biol Chem 2011;286 (11):9856-9864

85 Liu TF, Vachharajani VT, Yoza BK, McCall CE. NAD+-dependent sirtuin 1 and 6 proteins coordinate a switch from glucose to fatty acid oxidation during the acute inflammatory response. J Biol Chem 2012;287(31):25758-25769

86 Vachharajani VT, Liu T, Brown CM, et al. SIRT1 inhibition during the hypoinflammatory phenotype of sepsis enhances immunity and improves outcome. J Leukoc Biol 2014;96(05):785-796

87 Weavers H, Evans IR, Martin P, Wood W. Corpse engulfment generates a molecular memory that primes the macrophage inflammatory response. Cell 2016;165(07):1658-1671

88 Bekkering S, Quintin J, Joosten LAB, van der Meer JWM, Netea MG, Riksen NP. Oxidized low-density lipoprotein induces long-term proinflammatory cytokine production and foam cell formation via epigenetic reprogramming of monocytes. Arterioscler Thromb Vasc Biol 2014;34(08):1731-1738

89 Tripathi A, Debelius J, Brenner DA, et al. The gut-liver axis and the intersection with the microbiome. Nat Rev Gastroenterol Hepatol 2018;15(07):397-411

90 Samuel VT, Shulman GI. Nonalcoholic fatty liver disease as a nexus of metabolic and hepatic diseases. Cell Metab 2018;27 (01):22-41

91 Passeri MJ, Cinaroglu A, Gao C, Sadler KC. Hepatic steatosis in response to acute alcohol exposure in zebrafish requires sterol regulatory element binding protein activation. Hepatology 2009;49(02):443-452

92 Tang T, Sui Y, Lian M, Li Z, Hua J. Pro-inflammatory activated Kupffer cells by lipids induce hepatic NKT cells deficiency through activation-induced cell death. PLoS One 2013;8(12):e81949

93 Carthew RW, Sontheimer EJ. Origins and mechanisms of miRNAs and siRNAs. Cell 2009;136(04):642-655

94 Thomou T, Mori MA, Dreyfuss JM, et al. Adipose-derived circulating miRNAs regulate gene expression in other tissues. Nature 2017;542(7642):450-455

95 Korf H, van der Merwe S. Adipose-derived exosomal MicroRNAs orchestrate gene regulation in the liver: is this the missing link in nonalcoholic fatty liver disease? Hepatology 2017;66(05): 1689-1691

96 Ying W, Riopel M, Bandyopadhyay G, et al. Adipose tissue macrophage-derived exosomal miRNAs can modulate in vivo and in vitro insulin sensitivity. Cell 2017;171(02):372.e12-384. e12

97 Jiang X, Jiang L, Shan A, et al. Targeting hepatic miR-221/222 for therapeutic intervention of nonalcoholic steatohepatitis in mice. EBioMedicine 2018;37(October):307-321

98 Wang J, Leclercq I, Brymora JM, et al. Kupffer cells mediate leptininduced liver fibrosis. Gastroenterology 2009;137(02):713-723

99 Shen J, Sakaida I, Uchida K, Terai S, Okita K. Leptin enhances TNF$\alpha$ production via p38 and JNK MAPK in LPS-stimulated Kupffer cells. Life Sci 2005;77(13):1502-1515

100 Chatterjee S, Ganini D, Tokar EJ, et al. Leptin is key to peroxynitrite-mediated oxidative stress and Kupffer cell activation in experimental non-alcoholic steatohepatitis. J Hepatol 2013;58 (04):778-784

101 Imajo K, Fujita K, Yoneda M, et al. Hyperresponsivity to low-dose endotoxin during progression to nonalcoholic steatohepatitis is regulated by leptin-mediated signaling. Cell Metab 2012;16(01): 44-54

102 Xu A, Wang Y, Keshaw H, Xu LY, Lam KSL, Cooper GJS. The fatderived hormone adiponectin alleviates alcoholic and nonalcoholic fatty liver diseases in mice. J Clin Invest 2003;112(01): 91-100

103 Nagareddy PR, Kraakman M, Masters SL, et al. Adipose tissue macrophages promote myelopoiesis and monocytosis in obesity. Cell Metab 2014;19(05):821-835

104 Tomita K, Tamiya G, Ando S, et al. Tumour necrosis factor alpha signalling through activation of Kupffer cells plays an essential role in liver fibrosis of non-alcoholic steatohepatitis in mice. Gut 2006;55(03):415-424

105 Bijnen M, Josefs T, Cuijpers I, et al. Adipose tissue macrophages induce hepatic neutrophil recruitment and macrophage accumulation in mice. Gut 2018;67(07):1317-1327

106 Kishore P, Li W, Tonelli J, et al. Adipocyte-derived factors potentiate nutrient-induced production of plasminogen activator inhibitor-1 by macrophages. Sci Transl Med 2010;2(20): 20ra15

107 Halpern KB, Shenhav R, Massalha H, et al. Paired-cell sequencing enables spatial gene expression mapping of liver endothelial cells. Nat Biotechnol 2018;36(10):962-970

108 MacParland SA, Liu JC, Ma X-Z, et al. Single cell RNA sequencing of human liver reveals distinct intrahepatic macrophage populations. Nat Commun 2018;9(01):4383 